\title{
Radiopacity in the Bone Acrylic Cement based on the PMMA+HAp System Through Radiological Contrast Analysis
}

\author{
Carlos Julio Montaño ${ }^{1 *}$, Olga Lucia Maquilon², Luciana Batista Nogueira ${ }^{3}$, Sonia Seger Pereira \\ Mercedes $^{1}$ and Tarcísio Passos Ribeiro Campos ${ }^{1}$ \\ ${ }^{1}$ Universidade Federal de Minas Gerais - UFMG, Departamento de Engenharia Nuclear - Laboratorio de Radiações Ionizantes, Av. \\ Antônio Carlos, 6627, ZIP Code 31270-901, Belo Horizonte-MG / Brazil \\ ${ }^{2}$ Universidade Federal de Minas Gerais - UFMG, Departamento de Fisiologia e Biofísica - ICB - Laboratório de Biologia Vascular, Av. \\ Antônio Carlos, 6627, ZIP Code 31270-901, Belo Horizonte-MG / Brazil \\ ${ }^{3}$ Universidade Federal de Minas Gerais - UFMG, Departamento Anatomia e Imagem, Escola de Medicina, Av. Prof. Alfredo Balena, \\ 190 - sala 179, Belo Horizonte-MG / Brazil
}

Received 4 March 2020; Accepted 16 October 2021

\begin{abstract}
Vertebroplasty and Kyphoplasty have been studied for several years as percutaneous procedures for treatment of bone fractures, osteoporosis and other abnormalities. Currently, these procedures have been established as effective minimally invasive surgical procedures very promising in orthopedics and traumatology. Those procedures are guided by fluoroscopy using radiopaque substances such as barium sulfate $\left(\mathrm{BaSO}_{4}\right)$. To evaluate the bone cement based on the PMMA+HAp system as a radiopaque agent through deterministic analysis of radiological contrast. Vertebrae models in vitro was used as phantom to inject the implants All phantoms belong to a pig column piece. Four implants types was studied as radiological images: lead ( $\mathrm{Pb}$ 99.9\%), Polymethylmethacrylate (PMMA), 0.9PMMA+0.1BaSO $\mathrm{P}_{4}$ system, $0.5 \mathrm{PMMA}+0.5 \mathrm{HAp}$ system such that HAp (Hydroxyapatite). In the diagnosis radiology range, 0.5PMMA+0.5HAp system has a higher attenuation profile to difference of others implants based on acrylic. FWHM (Full Width at Half Maximum) was 2.19 by $0.5 \mathrm{PMMA}+0.5 \mathrm{HAp}$ system obtained with 3D-Gaussian surface fit. The 3D-Gaussian surface fit was obtained over graphs with FFT 2D (Fast Fourier Transform 2-Dimensional) arrays corresponding to ROI (Region of Interest) of each vertebral image. The $0.5 \mathrm{PMMA}+0.5 \mathrm{HAp}$ system used as bone cement produces a greater radiological contrast in the image when the HAp concentration achieves $50 \%$ in the bone cement. In present study, HAp plays an important role as radiopaque agent since it will allow suppressing or reducing other contrast agents.
\end{abstract}

Keywords: Bone cement, Contrast agents, Radiological contrast, Vertebroplasty

\section{Introduction}

An orthopedic procedure was developed in France in 1987 known as Vertebroplasty using an acrylic injected with a syringe inside the fractured vertebral body. In the surgical context, this procedure was known as a minimally invasive technique due to ambulatory nature. The technique was initially implemented for the vertebral hemangiomas treatment following this was applied to fractures for compression due to bone metastasis or osteoporosis. The PMMA (Polymethylmethacrylate with chemical nomenclature $\left.\left[\mathrm{CH}_{2} \mathrm{C}\left(\mathrm{CH}_{3}\right)\left(\mathrm{CO}_{2} \mathrm{CH}_{3}\right)\right]_{\mathrm{n}}\right)$ was used as an acrylic cement to join the broken bone pieces as part of percutaneous technique. The biggest problem of the percutaneous technique is extravasation of cement outside the bone region to be located. One solution to this undesirable event is the decompression into the vertebra by surgical reintervention [1-3]. These percutaneous procedures are complementary to therapeutic treatments within tumor control in the metastasis cases [4]. In most cases, patients with advanced metastatic disease stage receive palliative radiotherapy. Some studies have proposed IMRT (Intensity Modulated Radiotherapy) and IMR (Intensity-Modulated

*E-mail address: carlmont@ucm.es

ISSN: 1791-2377 @ 2021 School of Science, IHU. All rights reserved.

doi:10.25103/iestr.145.04
Radiosurgery) as therapeutic treatments with less neurological impact on the spinal cord. Although exists demyelinating phenomenon of nervous system due to high radiosensitivity because this is less likely to find adverse effects with the use of these radiotherapy techniques [5-8]. At 2006, other options have been proposed in the radiation therapy field based on the idealization of radioactive bone cement [9]. Such radioactive cement treats bone metastases in a way that eliminates the clonogenic properties in cancer cells along with the reinforcement of bone structure. At 2009, Quadramet ( ${ }^{153} \mathrm{Sm}$-EDTMP) administered percutaneously in the affected region by means of protocol Khyphoplasty (Kyphon) [10]. The variant incorporated in the percutaneous technique going to allow a balloon device to recover the anatomy for collapsed vertebra.

There are a number of biophosphates studied considered as bioceramics among them Hydroxyapatite (HAp). HAp has been one of the biophosphates often used in orthopedic procedures with chemical nomenclature $\left(\mathrm{Ca}_{5}\left(\mathrm{PO}_{4}\right)_{3} \mathrm{OH}\right)$. Because $69 \%$ of its composition is equivalent to bone in such osteoid, including up to $20 \%$ collagen fibers as connective tissue. Therefore, HAp is a fundamental part of natural bone. This compound has been proposed so that together with PMMA the mixture achieves an optimal adhesion response of the osteoblasts within the biomaterial [11-13]. 
Considering the bone implant, the improvement factors at quality guided-image is still necessary for Vertebroplasty technique through radiological contrast study. In this work, HAp shows benefits as radiopaque agent in the bone cement and the possibility of removing barium sulfate to reduce the toxicity. The contrast calculation was performed by evaluating the image attenuation profiles with the help of statistical and mathematical resources.

\section{Material and Methods}

\subsection{Anatomical sample preparation in-vitro}

A piece of pig vertebral column was delivery by a meat market in a special cut. That piece was embed in an equivalent muscle tissue in vitro, anatomically maintaining its structure disregarding others anthropometric characteristics on the model. The structure was immediately cooled to $-18{ }^{\circ} \mathrm{C}$ to reduce the effect of decomposition.

\subsection{Synthesis method and preparing acrylic bone cement} The HAp was synthesized by the sol-gel method. According to that technique, after mixing the components, the solution was left for 24 hours in a closed beaker to force the precipitate build up at the end of the reaction due to nucleation and formation of colloids finally [9, 14]. The compounds used for the synthesis of HAp were $3.937 \mathrm{~g}$ of $\mathrm{Ca}\left(\mathrm{NO}_{3}\right)_{2} \cdot 4 \mathrm{H}_{2} \mathrm{O}, 0.69 \mathrm{~mL}$ of $\mathrm{H}_{3} \mathrm{PO}_{4}, 2 \mathrm{~mL}$ of $\mathrm{CH}_{3} \mathrm{OH}$ and water as solvent in excess. Subsequently the sample was heated in an oven with ramp from room temperature to 100 ${ }^{\circ} \mathrm{C}$ for 22 hours as follows: from room temperature to $80{ }^{\circ} \mathrm{C}$ at a rate of $0.306{ }^{\circ} \mathrm{C} \cdot \mathrm{min}^{-1}$, then an isotherm 360 minutes, later to $100{ }^{\circ} \mathrm{C}$ a ramp at the rate of $0.333{ }^{\circ} \mathrm{C} \cdot \mathrm{min}^{-1}$ and finally an isotherm 720 minutes. The next day, the sample was heated from room temperature to $720{ }^{\circ} \mathrm{C}$ at a rate of 6 ${ }^{\circ} \mathrm{C} \cdot \mathrm{min}^{-1}$, then 60 minutes isotherm. The HAp powder is macerated and mixed in different proportions with the MMA (Methyl Ethyl Methacrylate $\left(\mathrm{C}_{5} \mathrm{H}_{8} \mathrm{O}_{2}\right)$ to produce $1 \mathrm{~g}$ of acrylic bone cement in a binary system (1-x) PMMA+xHAp, where $[\mathrm{x}]$ is the concentration of HAp in the mixture with $\mathrm{x}$ $=0,00$ and $\mathrm{x}=0,50$ for two implants.

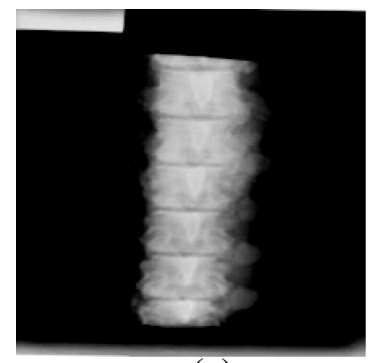

(a)

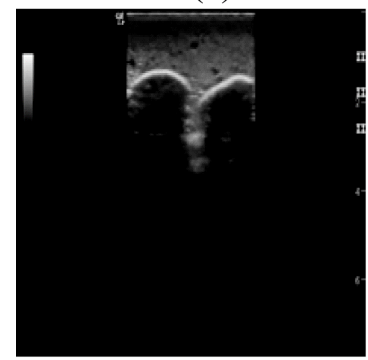

(c)

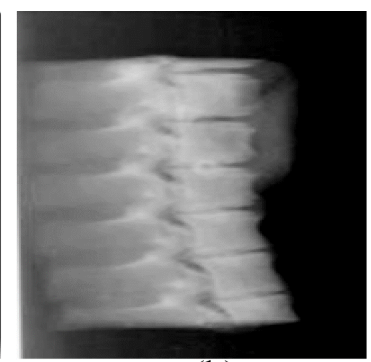

(b)

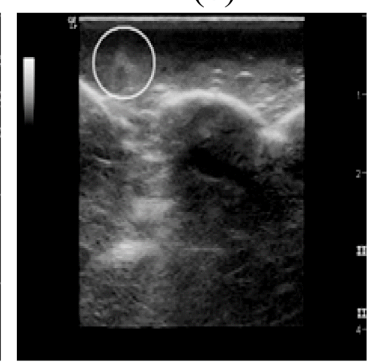

(d)
Fig. 1. X-ray and ultrasound images in vitro from vertebrae model. In (a) antero-posterior-AP incidence plane (b) lateral incidence plane (c) and (d), there are ultrasound images; and, (d), a dynamic anomaly shows the insertion of the needle into phantom.
The non-radioactive mixture was only prepared with PMMA, HAp, so that the dissolution held excess water as dissolvent. The solution in deionized water was done primarily to modulate the phases of the acrylic in order to increase the polymerization time by mean of stirring of the mixture. Both the PMMA and the instruments for making the mixture were also cooled due to the polymerization effect that is proportional to the temperature rise. Barium sulfate was mixed with PMMA following $0.9 \mathrm{PMMA}^{2} 0.1 \mathrm{BaSO}_{4}$ concentrations equation to perform another trial implant.

\subsection{Cement injection}

The cement was injected into vertebral bodies with an incline $45^{\circ}$ respect to the middle-sagittal plane and simulating a puncture procedure as if it was a Vertebroplasty kit. Four different implants were injected in an amount of 1 $\mathrm{g}, \mathrm{x}_{1}=\mathrm{Pb} 99,9 \%$ purity, $\mathrm{x}_{2}$ as PMMA acrylic implant, $\mathrm{x}_{3}=$ $0,9 \mathrm{PMMA}+0,1 \mathrm{BaSO}_{4}$ system and $\mathrm{x}_{4}=0,5 \mathrm{PMMA}+0,5 \mathrm{HAp}$ system. The Barium Sulfate $\left(\mathrm{BaSO}_{4}\right.$, stoichiometric nomenclature) incorporated in the $\mathrm{x}_{3}$ concentration as contrast agent simulating clinic radiological conditions.

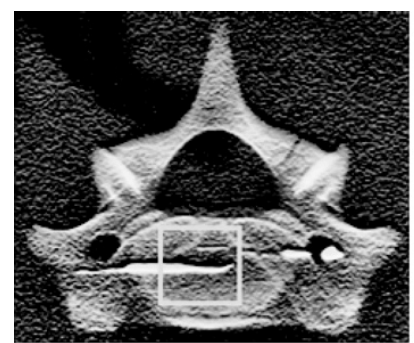

(a)

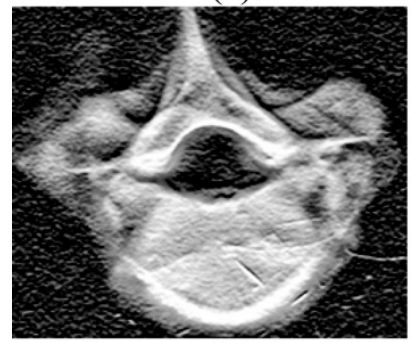

(c)

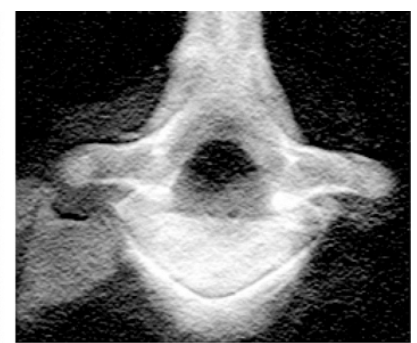

(b)

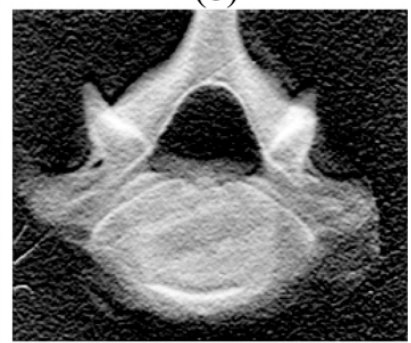

(d)
Fig. 2. Radiological images of four vertebral models. In (a) the vertebra have molten lead with ROI defined as 84 x 80 pixels on vertebral body (b) PMMA implant (c) $0.9 \mathrm{PMMA}^{2}+0.1 \mathrm{BaSO}_{4}$ implant. (d) $\mathrm{x}=0.5$ of the bone cement.

\subsection{Radiographic tests with $x$-ray equipment}

The X-ray equipment used was the BR 100, a mobile system with performance $100 \mathrm{~mA}$ and $90 \mathrm{kV}$. The BR 100 is composed with an X-ray tube sealed and linked to a telescope that is connected to the equipment column than have an arm that allows depending on the movement necessary adjustment of the distance between tube and film that is studied radiographically. It also has a setting control with all elements necessary to schedule the voltage and current intensity. According to the radiological techniques, exposure time of $0.50 \mathrm{~s}$, current of $70 \mathrm{~mA}$, voltage of $60 \mathrm{kV}$ and FFD (Focal Film Distance) of $85 \mathrm{~cm}$ were applied in thoracic vertebrae. Subsequently, films were scanning to study the radiological contrast in each of the vertebrae in which the bone cement was implanted in accordance with all of the above. A HP Scanjet G4050 transmission scanner was used to evaluate the radiological contrast in different points on the region of interest (ROI) at each image. 


\subsection{Ultrasonography images}

The GE Healthcare ultrasound equipment LOGIQe REF. 5199704 made in China in 2008 was used as helper equipment to assistance in the Vertebroplasty simulating procedure with the pig vertebral column. One 12L-RS probe was used in B-cine mode with a signal of $12 \mathrm{MHz}$ and with a maximum depth of $7 \mathrm{~cm}$ for the configuration abdominal type before to the application of the contact gel for pig column model in vitro. In the second image signal was 10 $\mathrm{MHz}$ and the field depth was defined to $4 \mathrm{~cm}$.

\section{Results}

\subsection{Physical distribution and attenuation profile of the implants}

The images obtained by mean x-ray equipment have good contrast even though the bone parts were embedded in a tissue simulating equivalent soft tissue. According to Fig 1(a), shown on antero-posterior (AP) incidence plane of the phantom, in which the X-ray beam was attenuated with $4 \mathrm{~cm}$ of Styrofoam this being phantom's container. The polystyrene and the soft equivalent tissue surrounding have a lower attenuation comparatively to bone tissues. Fig 1(b), a lateral image was shooting. Fig 1(c) and Fig 1(d), images provided by ultrasound equipment simulating the lumbar puncture protocol. In each image observed high quality definition on the spinous processes, transverse, upper, and lower joints. Just as the needle guided for the spinous processes in the lumbar puncture for patients in gestation, it is possible to make the same in Vertebroplasty. However, cement flow control has been better to make always with a fluoroscopy equipment.

The following images as shown in Fig 2 were taken from four vertebral bodies. Fig 2(a), one of them is observed with an inlay of lead in the linear shape that will be used as reference to evaluate the radiological contrast respect to another three images. Furthermore, the box in the vertebral body represents the ROI (Region of Interest) where the bone implant is likely to be found. In order to simplify the analysis of the contrast, the ROI size was located in the vertebral body. Fig 2(b) showed a vertebral body with a poorly differentiated image corresponding to case with PMMA implant. Fig 2(c), PMMA was injected with $10 \%$ $\mathrm{BaSO}_{4}$. In Fig. 2(d), polymerizing bone cement based on the (1-x)PMMA+xHAp binary system with a concentration [x], $\mathrm{x}=0.5$ was injected. The bone cement is well spread in the bone piece and with better contrasts. The most important feature of this test was to find a relatively differentiated image despite to suppress $\mathrm{BaSO}_{4}$ in the bone cement.

Fig 3 shows the implant in one of the vertebrae and the image treatment on the implant region. The implant region can be understood as a function of gray levels along a given length. Furthermore, attenuation profile showed for each implant material corresponds to a qualitatively measure. Fig 3(a) show the changes produced in terms of the incorporated implant in the bone piece with lead. Fig 3(b) show the region of the perforation and its implant in a high relief plane. In an image of 8-bits, the scale corresponds to 256 gray levels, such that, the black represents zero and white to 255 value as showed in Fig 3(c) for lead implant. The maximum differentiation on the image among these two integer values is physically related to radiation attenuation in the radiological case [15]. For such vertebral body, Fig 3(d) shows gray level histograms along a linear distance with 62 pixels at the implant site. The gray levels found in Fig 3(d) for the two 0.9PMMA+0,1 $\mathrm{BaSO}_{4}$ and $0,5 \mathrm{PMMA}+0,5 \mathrm{HAp}$ samples behaved very similarly. At the last, the gray levels for PMMA without contrast exhibit less difference with the image background. The qualitative analysis carried out inferred about the attenuation of the x-ray field according to each one of the implants used in the experiment. The attenuation is greater for constituents with high atomic numbers such as lead $(\mathrm{Pb})$ defining this as a positive control in the experiment. In the case of the $0,5 \mathrm{PMMA}+0,5 \mathrm{HAp}$ system compared to other substances, the attenuation factor corresponds to a linear combination of the attenuation factors for all the fundamental constituent elements. On the other hand, x-ray beam attenuation in the radiological diagnostic domain physically follow the superposition principle as described below: $(\mu / \rho)_{T}=\sum_{i=0}^{i=n} w_{i}(\mu / \rho)_{i}$. Such that $w_{i}$ is the attenuation's weight of each element in the compound. Fig 3(e) shows the attenuation spectrum of the photons with energies in the diagnosis range. This energy range corresponding to the x-ray field used in the experiments allows confirming to lead as a substance with highest attenuation. Likewise, the bone cement proposed as an implant has an attenuation factor close to the PMMA with contrast agent.

\subsection{Radiological contrast}

The radiological contrast can be quantified based on the attenuation profile of gray levels for each collection of points in space $(\mathrm{x}, \mathrm{y})$, shown in Fig 4. The complexity on the image analysis can be reduced using a specific ROI, equivalent to all images with same size. The mathematical elements indicate that the ROI is defined topologically as an area for all the points $(\mathrm{x}, \mathrm{y})$ where probably exists the implant which is associated with a set of gray intensities $\left(k_{x}, k_{y}\right)$. Each point in our image $I(x, y) \curvearrowright \tilde{I}\left(k_{x}, k_{y}\right)$ can be transformed to the frequencies space associated with the gray levels. On the other hand, the number of gray levels is adjusted computationally to the weight of the image determined by the configuration established in the scanning device.

In every analysis can be changed the size of the image after the scanning procedure and as result a significant loss of resolution would be likely. The computational size of the images was configured to 8-bits. Therefore, the images were digitally reconstructed with 256 levels of gray. If each points profile in the spatial domain is treated as a function then such math behavior could be considered bounded, monotonous and periodic. Therefore, the definition by Suetens for the contrast of an image would be mathematically congruent from this point of view [15]. An arrays set was generated by mean FFT 2D (Fast Fourier Transform in two dimensions) calculation for each ROI. The surfaces referent to those arrays, corresponding to amplitude in the Fourier domain for spatial frequency, such graphs were presented in Fig 4. The Figs 4(b), 4(c), 4(d), 4(e) correspond to the FFT 2D of the ROI defined in Fig 2 in each vertebra. The surfaces represent gray levels space that make up the image. In this way, considering the definition made by Suetens for the contrast of an image [15], the FFT 2D corresponds to:

$$
\begin{aligned}
& \tilde{I}(\vec{k})=\frac{1}{\sqrt{2 \pi}} \int_{-\infty}^{+\infty} d \vec{r} e^{-i \vec{k} \cdot \vec{r}} I(\vec{r}) \\
& I(\vec{r})=\frac{1}{\sqrt{2 \pi}} \int_{-\infty}^{+\infty} d \vec{k} e^{i \vec{k} \cdot \vec{r}} \tilde{I}(\vec{k})
\end{aligned}
$$


Eq. 2 represents the inverse transform that is defined as a characteristic function from all constituents points in the image space, such that $\vec{r} \rightarrow(x, y)$ and $\vec{k} \rightarrow\left(k_{x}, k_{y}\right)$ where $\vec{k}$ is the space of spatial frequencies. In addition, $I(\vec{r})$ also depends about this attenuation profile of the x-ray field that impacts the radiographic film and that will be attenuated by mean the vertebra and bone implant.

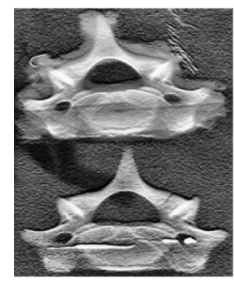

(a)

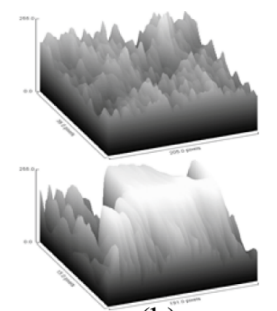

(b)

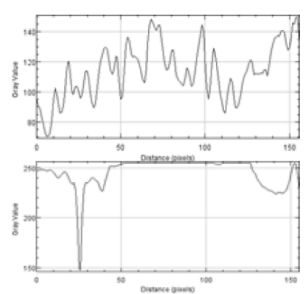

(c)

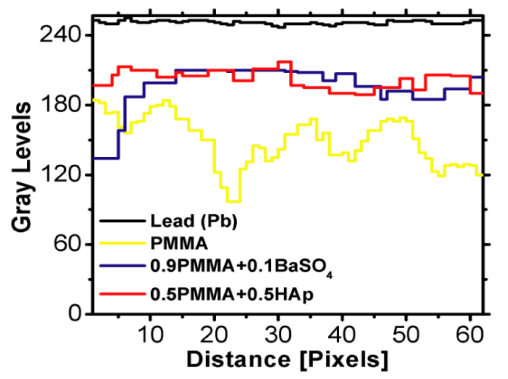

(d)

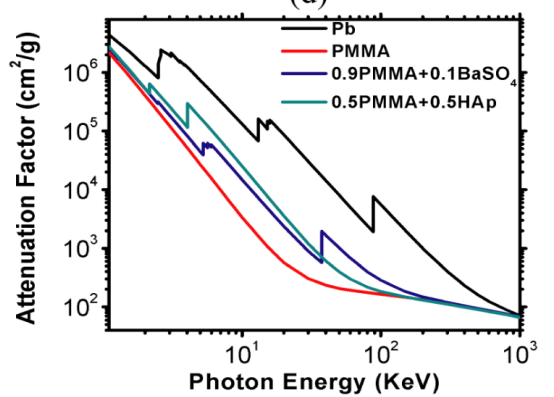

(e)

Fig. 3. Gray level histograms from implants for every vertebral phantom and attenuation profile. (a) and (b) implant's surface plot and the vertebra image has molten lead and with the hole, respectively. (c) Gray level histograms of the phantom with the lead implant and other with its hole, respectively. (d) Gray level histograms from a plots line for different implants to a length equivalent with 62 pixels. (e) Attenuation factor for all implants download from NIST, online free XCOM. (https://physics.nist.gov/PhysRefData/Xcom/html/xcom1.html).

In Figs 5(a), 5(b), 5(c), 5(d), the 3D-surfaces plot for such FFT 2D array were obtained by mean the software Origin 2019b. These surfaces are defined as a subspace of points $S=S\left(k_{x}, k_{y}\right)$ in the Fourier domain. The 3D-Gaussian fit was better mathematical approximation to these surfaces corresponding to FFT 2D arrays. Fig 5(e) shows the FFT 2D amplitude graph and 3D-Gaussian fitting for ROI with the lead implant. The software made the best fitting through a certain iterations number. The user could define the Gaussian fitting parameters, reducing the standard error. Tab. 1 presents a summary for the four implants of different types used in every vertebra studied.

\section{Discussion}

The FWHM (Full Width at Half Maximum) was calculated on the 3D-Gaussian surface fit considering all the statistical parameters. This measure is proportional to radiological contrast of ROI for each image studied here. The characteristic values related to all points that form 3DGaussian surface fitting were considered in the image analysis with a computational 8-bits weight. The values in Table 1 showed a great contrast in the ROI with the $P b$ implant as consequence of its high attenuation factor, thus contributing greatly to the heterogeneity of the image. Such result was taken as reference in all experiments. The contrast of the bone cement of PMMA+HAp was compared to the polymer coupled with contrast medium $\mathrm{PMMA}+\mathrm{BaSO}_{4}$ such that a greater amount of HAp in the bone cement can be equivalent to the radiopacity in the $\mathrm{PMMA}+\mathrm{BaSO}_{4}$ acrylic cement proposed by Boolen et al. [16].

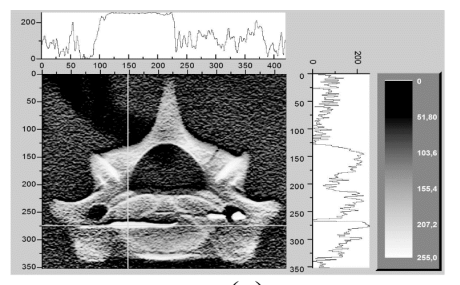

(a)

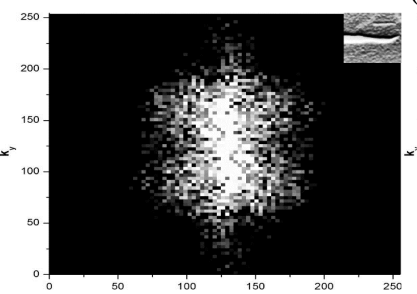

(b) ${ }^{k_{*}}$

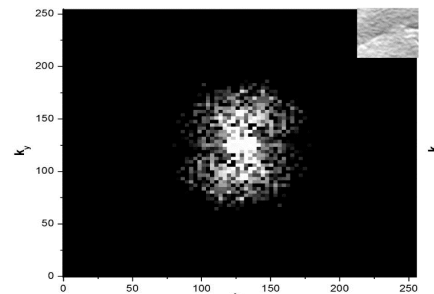

$(d)^{k_{*}}$

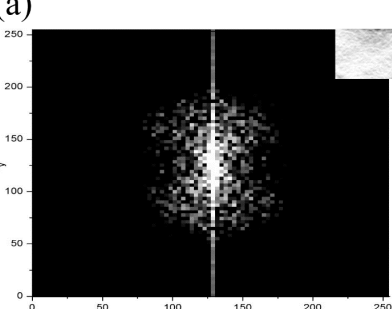

(c) ${ }^{100}$

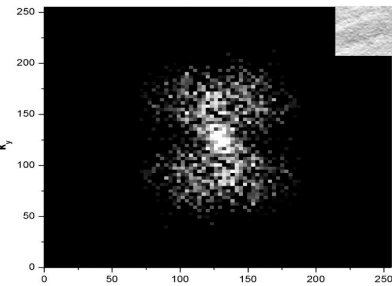

(e)
Fig. 4. The gray-profiles in the spatial domain of the $I(x, y)$ and FFT $2 \mathrm{D}$ images of their respective ROIs. Example for a gray profile in (a) straight lines parallel to $\mathrm{x}=0$ and $\mathrm{y}=0$; (b) FFT 2D for ROI with the lead implant; (c) FFT 2D for ROI with PMMA implant; (d) FFT 2D for ROI with the 0.9PMMA $+0.1 \mathrm{BaSO}_{4}$ implant; and, (e) FFT 2D for ROI with PMMA+HAp implant and 1:1 ratio.

Currently, Vertebroplasty procedure needs bone cements based on acrylics and according to the protocols, these commercial cements have a maximum of $20 \%$ of biophosphates because their use is focused on joining and stabilizing fractured bone pieces. The 0.5PMMA+0.5HAp system studied here from the radiological perspective consists in $50 \%$ biophosphates. This concentration has been proposed for other therapeutic applications e.g. Radiovertebroplasty [17].

The calculation of the radiological contrast in this work was carried out from a deterministic perspective because it assume the existence of an implant in the vertebral body region. In other radiological imaging studies, mainly associated with medical diagnosis, changes in contrast are suggestive to pathology and therefore associated with a disease. Therefore, in the case of medical diagnosis, contrast analysis should be assessed with a mainly stochastic approach, such as the diagnosis of diseases associated with 
cancer. Stochastic algorithms methods are added to optimize the computational analysis showing results with greater precision.

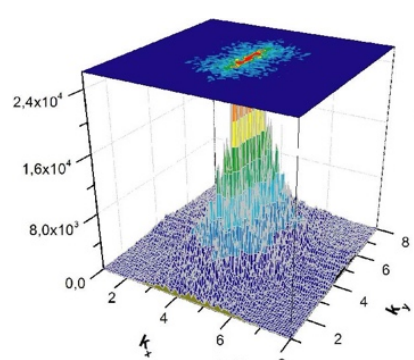

(a)

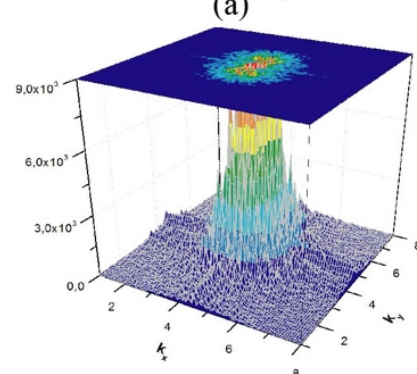

(c)

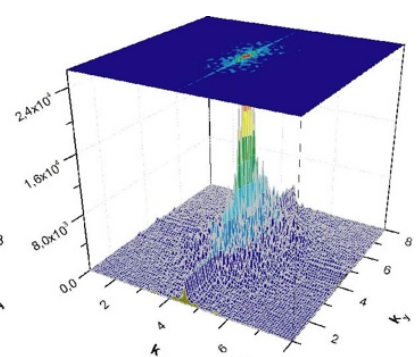

(b)

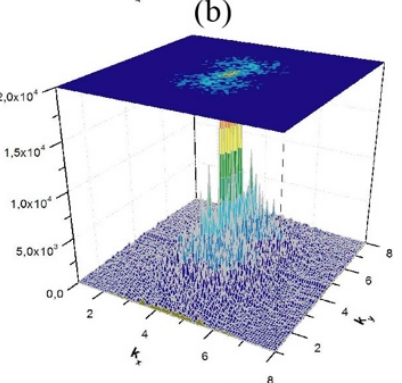

(d)

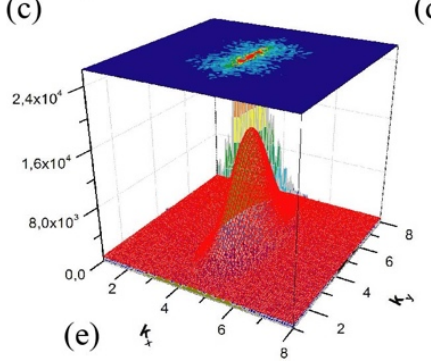

Fig. 5. FFT 2D curves from ROIs and Gauss surface fit. (a) Plotting Surface of the FFT 2D corresponds to ROI with lead implant. (b) Plotting Surface of the FFT 2D corresponds to ROI with PMMA implant. (c)Plotting Surface of the FFT 2D corresponds to ROI with the $0.9 \mathrm{PMMA}^{2} 0.1 \mathrm{BaSO}_{4}$ implant (d) Plotting Surface of the FFT 2D corresponds to ROI with PMMA+HAp implant, and 1:1 ratio. (e) FFT 2D graph and 3D-Gauss surface fit for the lead implant.

Table 1. Evaluation of the Radiological Contrast through FWHM due to the implants.

\begin{tabular}{|c|c|c|c|c|c|c|}
\hline Testing implants & $\begin{array}{c}\text { Standar } \\
\text { d } \\
\text { Deviatio } \\
\text { n } \sigma_{x}\end{array}$ & $\begin{array}{c}\text { Error } \\
\Delta \sigma_{x}\end{array}$ & 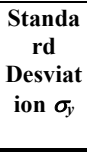 & $\begin{array}{l}\text { Erro } \\
\text { r } \Delta \sigma_{y}\end{array}$ & $\begin{array}{c}\text { *SD } \\
\sigma \\
\Delta \sigma= \\
\pm 0,0 \\
1 \\
\end{array}$ & $\begin{array}{c}\text { Radiologic } \\
\text { al } \\
\text { Contrast } \\
\text { FWHM } \\
\pm 0,02 \\
\end{array}$ \\
\hline Lead - Pb & 0,425 & 0,005 & 1,11 & 0,01 & 1,18 & 2,79 \\
\hline PMMA & 0,232 & 0,003 & 0,83 & 0,01 & 0,86 & 2,04 \\
\hline $\begin{array}{l}\text { 0.9PMMA+0.1BaS } \\
\mathrm{O}_{4}\end{array}$ & 0,418 & 0,005 & 0,81 & 0,01 & 0,91 & 2,14 \\
\hline $\begin{array}{l}\text { 0.5PMМА+0.5HA } \\
\mathrm{p}\end{array}$ & 0,345 & 0,005 & 0,86 & 0,01 & 0,93 & 2,19 \\
\hline
\end{tabular}

$\sigma^{2}=\sigma_{x}^{2}+\sigma_{y}^{2} ; F W H M=2 \sigma \sqrt{2 \operatorname{Ln} 2}$
The spatial distribution of this material was observed through radiological images obtained after cement application that show the possibility of incorporating an adequate amount of cement mass in the bone structure. Percutaneous column procedures guided by fluoroscopy have been already proposed [18, 19]. The radiopaque components nature were expressed on the radiological analysis from bone cement. After cross cutting in some of the vertebrae, the compound was solidified next being injected. In addition, the spreading of the material in bone tissue was larger in the spongy bone tissue. For clinic experience, there is important to reduce toxicity suppressing as much as possible the substances, which are not completely biocompatible with organic tissues, as radiopaque compounds used in medical imaging. In the bone microenvironmental, the high cement porosity allows phenomenon as migration of bone cells inside the implant. [20, 21]. Likewise, a highly porous bone cement allows greater diffusion of bone marrow, vascularization (angiogenesis) and cellular adhesion [22-25]. An increase in the HAp concentration for bone cement becomes a determining factor of the above.

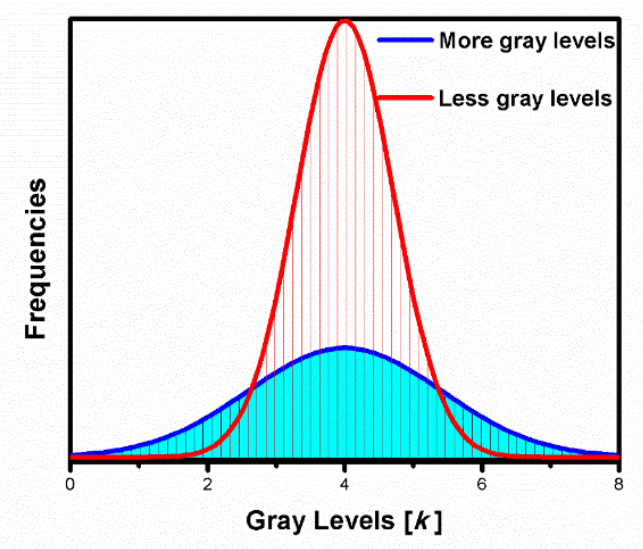

Fig. 6. Fourier sfor gray levels or $k$ numbers. More gray levels, highly differentiated image or less gray levels, poorly differentiated image.

\section{Acknowledgements}

The authors are grateful for the financial support from the CAPES, REBRAT-SUS project supported by CNPq. The corresponding author thanks the OEA-GCUB 2014 program for the opportunity offered with the doctoral scholarship in this academic and research experience.

This is an Open Access article distributed under the terms of the Creative Commons Attribution License.

\section{Conclusion}

\section{References}

1. G. Chen, Z.-P. Luo, H. Zhang, B. Nalajala, H. Yang. Journal of Clinical Neuroscience 20, 948-950 (2013).

2. B. Emami, J. Lyman, A. Brown, L. Cola, M. Goitein, J. Munzenrider, B. Shank, L. Solin, M. Wesson. International Journal of Radiation Oncology* Biology* Physics 21, 109-122 (1991).
3. P. C. Gerszten, Orthopedic Clinics of North America 38, 441-450 (2007).

4. F. R. Hendrickson, W. M. Shehata, A. B. Kirchner. International Journal of Radiation Oncology* Biology* Physics 1, 275-278 (1976). 
Carlos Julio Montaño, Olga Lucia Maquilon, Luciana Batista Nogueira, Sonia Seger Pereira Mercedes and Tarcísio Passos Ribeiro Campos/Journal of Engineering Science and Technology Review 14 (5) (2021) 30 - 35

5. T. Kaneko, V. Sehgal, H. Skinner, M. Al-Ghazi, N. Ramisinghani, J. Keyak. Physics in Medicine \& Biology 55, 2451 (2010).

6. S. Ryu, F. Fang Yin, J. Rock, J. Zhu, A. Chu, E. Kagan, L. Rogers, M. Ajlouni, M. Rosenblum, J. H. Kim. Cancer: Interdisciplinary International Journal of the American Cancer Society 97, 20132018 (2003).

7. S. Ryu, J. Y. Jin, R. Jin, J. Rock, M. Ajlouni, B. Movsas, M. Rosenblum, J. H. Kim. Cancer: Interdisciplinary International Journal of the American Cancer Society 109, 628-636 (2007).

8. Y. Yamada, M. H. Bilsky, D. M. Lovelock, E. S. Venkatraman, S. Toner, J. Johnson, J. Zatcky, M. J. Zelefsky, Z. Fuks. International Journal of Radiation Oncology* Biology* Physics 71, 484-490 (2008).

9. T. P. R. Campos, R. D. A. Macedo, I. N. d. P. Industrial, Ed. (Brasil, 2006), vol. PI 0605721-7 A2.

10. H. Ashamalla, E. Cardoso, M. Macedon, A. Guirguis, L. Weng, S. Ali, B. Mokhtar, M. Ashamalla, N. Panigrahi. International Journal of Radiation Oncology* Biology* Physics 75, 836-842 (2009).

11. S. Dorozhkin, Materials 2, 399-498 (2009).

12. S. V. Dorozhkin, Biomaterials 31, 1465-1485 (2010).

13. A. E. Hirsch, B. S. Rosenstein, D. C. Medich, C. B. Martel, J. A. Hirsch, Pain Physician 12, 887-891 (2009).

14. R. LeGeros, S. Lin, R. Rohanizadeh, D. Mijares, J. LeGeros, Journal of materials science: Materials in Medicine 14, 201-209 (2003).

15. P. Suetens, Fundamentals of medical imaging. (Cambridge university press, 2017)
16. E. J. Boelen, G. Lewis, J. Xu, T. Slots, L. H. Koole, C. S. van Hooy-Corstjens. Journal of Biomedical Materials Research Part A: An Official Journal of The Society for Biomaterials, The Japanese Society for Biomaterials, and The Australian Society for Biomaterials and the Korean Society for Biomaterials 86, 76-88 (2008).

17. C. J. Montaño, T. P. R. d. Campos, Acta ortopedica brasileira 27, 64-68 (2019).

18. A. E. Hirsch, D. C. Medich, B. S. Rosenstein, C. B. Martel, J. A. Hirsch, Radiotherapy and Oncology 87, 119-126 (2008).

19. T. M. Turner, R. M. Urban, K. Singh, D. J. Hall, S. M. Renner, T.H. Lim, M. J. Tomlinson, H. S. An, The Spine Journal 8, 482-487 (2008).

20. K. Agrawal, G. Singh, D. Puri, S. Prakash, Journal of Minerals and Materials Characterization and Engineering 10, 727 (2011).

21. W. Suchanek, M. Yoshimura, Journal of Materials Research 13, 94117 (1998).

22. G. Daculsi, O. Laboux, O. Malard, P. Weiss, Journal of Materials Science: Materials in Medicine 14, 195-200 (2003).

23. M. Dalby, L. Di Silvio, E. Harper, W. Bonfield, Biomaterials 22, 1739-1747 (2001).

24. M. Dalby, L. Di Silvio, E. Harper, W. Bonfield, Biomaterials 23, 569-576 (2002).

25. S. Yamada, D. Heymann, J.-M. Bouler, G. Daculsi, Biomaterials 18, 1037-1041 (1997). 